\title{
Postkoloniale und postimperiale Ansätze in der Imperiumsforschung
}

\begin{abstract}
Tagungsbericht: "Habsburg Postcolonial \& Beyond. Postkoloniale und postimperiale Forschungskonzepte in den Kulturwissenschaften « (Zagreb, 17.-19.3.2016)
\end{abstract}

Ab Juni 2015 läuft an der Abteilung für Germanistik der Philosophischen Fakultät der Universität Zagreb ein kultur- und literaturwissenschaftliches Forschungsprojekt unter dem Titel »Postimperiale Narrative in den zentraleuropäischen Literaturen der Moderne $« .{ }^{1}$ Als Ausgangsposition des Projektes sind Berührungspunkte in der Konstruktion kultureller und literarischer Strukturen im postimperialen Raum der Habsburger Monarchie gedacht, wobei vor allem der für den Einbruch der Moderne kennzeichnende Prozess der Nationsbildung stark in den Blick genommen wird. Der Begriff des (Post-) Imperialen weist dabei auf ein komplexes Netzwerk von Wechselwirkungen zwischen dem (ehemaligen) imperialen Zentrum und dessen Peripherie hin. Das Forschungsteam, in dem WissenschaftlerInnen aus Dublin, Ljubljana, Köln, Szeged, Wien und Zagreb zusammenarbeiten, untersucht in erster Linie das Erbe der Donaumonarchie und seinen nachhaltigen Einfluss auf den gemeinsamen, über- und transnationalen kulturellen Raum.

Die Ergebnisse der Forschungsarbeit im ersten Projektjahr wurden vom 17. bis 19. März 2016 an der Philosophischen Fakultät in Zagreb im Rahmen der Tagung »Habsburg Postcolonial \& Beyond. Postkoloniale und postimperiale Forschungskonzepte in den Kulturwissenschaften « vorgestellt. Teilgenommen haben insgesamt 18 ForscherInnen aus In- und Ausland, die teils theoriebezogene Vorträge, teils Fallstudien präsentierten.

1 Das Forschungsprojekt »Postimperiale Narrative in zentraleuropäischen Literaturen der Moderne « wird von der Kroatischen Wissenschaftsstiftung (HRZZ) finanziert (Ref.-Nr. IP-201409-2307 POSTIMPERIAL) und unter der Leitung von Marijan Bobinac durchgeführt. Für weitere Informationen (Projektbeschreibung, MitarbeiterInnen, Aktivitäten usw.) s. <http:// postimpnarrative.ffzg.unizg.hr>. 
Als Einführung in die Tagung sowie in die andauernde und erfolgreiche Zusammenarbeit Zagreber GermanistInnen mit WissenschaftlerInnen aus Wien, Sarajevo und Dublin, die schließlich zum aktuellen Forschungsprojekt führte, wurden am 17. März 2016 im Österreichischen Kulturforum drei mit dem Forschungsthema eng verbundene Sammelbände präsentiert: »The Long Shots of Sarajevo "1914-2014 (Hgg. Vahidin Preljević, Clemens Ruthner. Tübingen: Francke 2016); Sarajevski dugi pucnji 1914. (Hgg. Vahidin Preljević, Clemens Ruthner. Zenica: Vrijeme 2016) und Wechsel Wirkungen. Austria-Hungary, Bosnia-Herzegovina, and the Western Balkans, 1878-1918 (Hgg. Clemens Ruthner et al. New York: Peter Lang 2015). Bei den ersten zwei Titeln handelt es sich um die Proceedings der gleichnamigen interdisziplinären Konferenz, die anlässlich des 100. Jahrestags des Attentats in Sarajevo ebenda vom 24. bis 28. Juni 2014 stattgefunden hatte. Während die deutsche und bosnische Version des Sammelbandes von Milka Car vorgestellt wurden, gab Marijan Bobinac einen Überblick über den Sammelband Wechselwirkungen, worauf die Herausgeber, Vahidin Preljević (Universität Sarajevo) und Clemens Ruthner (Trinity College Dublin), das Wort ergriffen. Preljević und Ruthner schilderten das prekäre gesellschaftliche und politische Klima in Bosnien und Herzegowina, das nicht nur die Entwicklung des Landes während und im Nachfeld der Habsburger Monarchie prägte, sondern in Langzeitwirkung - aufgrund einer schwierigen Erinnerungskultur - auch die Organisation der oben erwähnten Konferenz sowie die Veröffentlichung der Sammelbände beträchtlich erschwerte. Dabei galt das Augenmerk im Besonderen dem historischen Status Bosniens innerhalb der Habsburger Monarchie sowie der Frage nach dem inneren Kolonialismus in der Donaumonarchie.

Der wissenschaftliche Teil des Symposiums wurde am 18. März in der Bibliothek der Philosophischen Fakultät in Zagreb eröffnet. In seinem Einleitungswort schilderte Marijan Bobinac (Universität Zagreb) als Leiter des Forschungsprojekts kurz Forschungsthema und methodologische Grundlagen. Auf der einen Seite wird die Projektmethodologie dem Forschungsparadigma $>$ Habsburg postcolonial entnommen, das in Anlehnung an angelsächsische postkoloniale Studien die komplexe Verschränkung von Sprache, Kultur und Politik in der Monarchie untersucht. Auf der anderen Seite entstammt der Begriff des >Imperiums` den geopolitischen Studien von Dominic Lieven und Herfried Münkler, wie auch der transnationalen Historiografie von Jürgen Osterhammel. Im Kontext eines solchen, kulturwissenschaftlich breit angelegten Forschungsparadigmas sollen stereotype, polarisierte Vorstellungen von der Habsburger Monarchie destabilisiert werden: Aus der Perspektive des Projektes sollte die Donaumonarchie, so 
Bobinac, weder als >Völkerkerker $\triangleleft$, noch als eine idealisierte, multinationale `Einheit in der Vielfalt‘, sondern eher als ein komplexer, oft durch Paradoxa gekennzeichneter kultureller Raum bezeichnet werden.

Nach dem Einleitungswort trat einer der Pioniere des Forschungsansatzes `Habsburg postcolonial selber auf. In seinem Keynote-Vortrag unter dem Titel K.u.k. post/kolonial oder post/imperial thematisierte Clemens Ruthner die im Titel der Konferenz angekündigte Fragestellung nach der angemessenen Methodologie bei der Untersuchung der Donaumonarchie. Am Anfang wurde konstatiert, dass der Begriff `Postimperialismus in der Politikwissenschaft dem Begriff `Kolonialismus übergeordnet ist. Dieser terminologische Unterschied kommt, so Ruthner, insbesondere dann zum Vorschein, wenn im Diskurs über den Kolonialismus zwischen äußerem (Überseekolonialismus) und innerem (`kontinentalem`) Kolonialismus unterschieden wird. In diesem Sinne ist Ruthners Anliegen eine Dekonstruktion dieser binären Opposition, die auf der Dyade zwischen `Eigenem ‘ und >Fremdem $>$ beruht. Dabei erinnerte er daran, dass die Opposition zwischen dem `Selbst $\triangleleft$ und dem >Anderem`schon bei Julia Kristeva, bzw. in ihrer Lektüre Sigmund Freuds, demontiert wird, und stellte schließlich die Frage, ob sich die Kulturwissenschaft der Produktivität halber nicht eher einem auf Ähnlichkeit statt auf Differenz beruhenden Ansatz zuwenden sollte.

Für einen Paradigmenwechsel von postkolonialen zu postimperialen Studien setzte sich auch Wolfgang Müller-Funk (Universität Wien) in seinem Vortrag Von den postcolonial studies zu den post-imperialen Studien ein. Im ersten Teil seines Beitrags wurde aufgrund von Schmidts Unterscheidung zwischen fluiden und territorialen Mächten festgestellt, dass `Kolonialismus und \Imperialismus keine deckungsgleichen Termini sind. Aufbauend auf Schmidts Theorie sieht Müller-Funk zwischen den postimperialen und den postkolonialen Ansätzen einen diskursgeschichtlichen Unterschied: Auf der einen Seite wird im postimperialen Ansatz, welcher der Geschichte und Zeitgeschichte entlehnt wird, der Imperiumsbegriff neutral verwendet. Auf der anderen Seite steht derselbe Begriff im Rahmen postkolonialer Studien für das Instrument kolonialer Herrschaft und Ausbeutung indigener Bevölkerung, weswegen er prinzipiell mit negativen Konnotationen in Verbindung gebracht wird. Für Müller-Funk besteht der analytische Gewinn postimperialer Forschungsansätze in der genauen Differenzierung unterschiedlicher, häufig synonym verwendeter Begriffe (Kolonialismus, Postkolonialismus, Imperialismus), wobei die moderne Nation als »Gegenspielerin des Imperialen « verstanden wird. Die Anwendbarkeit seines theoretischen Modells wurde durch eine kurze Analyse zweier Texte von Adam Bodor und Joseph Roth veranschaulicht. 
Der Frage nach dem adäquaten theoretischen Zugang zu postimperialen Narrativen wurde auch von Christine Magerski in ihrem Vortrag Mission im/ possible. Zur Reichweite imperialer Semantik nachgegangen. Als eine mögliche Lösung dieser methodologischen Frage wurde von Magerski der theoretische Ansatz des deutschen Politologen und Historiografen Herfried Münkler vorgeschlagen. Wie bekannt, gilt für Münkler die Habsburger Monarchie aufgrund ungenügender territorialer Expansion nicht als Weltreich. Wegen seiner langen zeitlichen Dauer schreibt Münkler der Donaumonarchie dennoch den Status eines Großreichs zu. Wie Magerski in ihrem Vortrag deutlich machte, erfüllen Imperien bei Münkler die Funktion der Herstellung von Ordnung sowohl auf geopolitischer als auch auf symbolischer Ebene. Dementsprechend fokussierte sie die symbolische Dimension des Imperialen, bzw. den Begriff der imperialen Mission als einer kosmologischen Rechtfertigung für die Existenz der Welt. Dabei wird als zentrales analytisches Konzept der Mythos in den Vordergrund gerückt, und zwar nicht nur in Bezug auf die ideologische Rechtfertigung des Imperialen, sondern auch in Bezug auf das antimythische Potential postimperialer Literatur.

Im darauffolgenden Vortrag von Christian Kirchmeier (Ludwig-Maximilians-Universität München) wurden anhand literarischer Beispiele drei Typen politischer Fremdheit herausgearbeitet. Im ersten Text - Reise um die Welt (1778/80) von Georg Forster - wurde der Fremdheitstypus der Alienität analysiert. Die Alienität beruht nach Kirchmeier auf dem Paradox eines transzendenten Raums, dessen Grenze zur Erfahrungswelt nur durch den Fremden überschritten werden kann. Den zweiten Fremdheitstypus die Alterität - analysierte Kirchmeier am Beispiel von Max Frischs Homo faber (1957). Im Kontext der Alterität wird der Fremde als ein heimatloser, permanent dislozierter Grenzgänger und `alter ego ‘ definiert. Das dritte literarische Beispiel - der berühmte Roman Radetzkymarsch (1932) von Joseph Roth - diente zur Veranschaulichung des dritten Fremdheitstypus: der Liminalität. Dabei wohnt dem Begriff der Liminalität bei Kirchmeier eine gleichzeitige Ein- und Ausgeschlossenheit aus dem gemeinsamen (imperialen) Raum inne, die vom besonderen Interesse für das Forschungsprojekt ist.

Mit dem Begriff der Liminalität und der damit verbundenen Spannung zwischen dem imperialen Zentrum und der dazugehörigen Peripherie wurde der thematische Übergang zum Thema der Raumforschung markiert. Der erste Vortrag zu diesem Thema - Urbane Topografien des (Post-)Imperialen. Bilanz und Ausblick aus ungarischer Sicht - wurde von Endre Hárs (Universität Szeged) gehalten. Hárs beleuchtete die Urbanisierung in Ungarn in der postimperialen Epoche, wobei die Stadt als der zentrale Begriff für die Wahrnehmung der Moderne hervorgehoben wird. Ausgehend von der 
zentralen Stellung der Stadt für die ungarische postimperiale Entwicklung wurde die routinierte Gegenüberstellung von Wien und Budapest als analytisches Verfahren der Kritik unterzogen, weil dieser seit den 1980er Jahren aktuelle Diskurs schon klischeehafte Züge annimmt. Dem setzte Hárs die Perspektive literarischer Topografien postimperialer Räume entgegen.

Die historiografische Perspektive auf die Raumforschung lieferte Drago Roksandić (Universität Zagreb) im Vortrag Imperial Multiple Borderlands' Concept and the Early Modern Croatian History, in dem das ab 1996 laufende Forschungsprojekt »Triplex Confinuum. Die Militärgrenze in der modernen Historiografie» vorgestellt wurde. Im Projekt wird die im 16. Jahrhundert zum Schutz der Habsburger Monarchie vor dem Osmanischen Reich etablierte militärische Zone in Slawonien und dem Banat (Kroatien) untersucht. Roksandić zeigte in seinem Vortrag, dass das Schutzgebiet in Kroatien als Kontaktzone zwischen dem Osmanischen Reich, der Donaumonarchie und der Republik Venedig als Musterbeispiel imperialer Raumkonstruktion fungiert. Überdies wurde veranschaulicht, wie die im Rahmen des Projektes erworbenen wissenschaftlichen Erkenntnisse an der Abteilung für Geschichte im Unterricht eingesetzt werden.

Eine weitere Perspektive auf die imperiale Peripherie wurde von Svjetlan Lacko Vidulić (Universität Zagreb) angeboten, der den Reisebericht Dalmatinische Reise (1909) von Hermann Bahr in Bezug auf seinen Vorschlag für die Lösung der `slawischen Frage ‘ analysierte. Lacko Vidulić zeigte, dass der Reisebericht die imagologische Wahrnehmung der Peripherie aus der Perspektive des Zentrums auf den ersten Blick übersteigt, da sich Bahr für einen föderalistischen Staat einsetzt. Die Befürwortung der föderalistischen Lösung kann jedoch einem genaueren Blick nicht standhalten, denn im kulturellen und symbolischen Bereich sollte, in Bahrs Perspektive, nach wie vor der österreichische Einfluss bewahrt werden. Infolgedessen wurde von Lacko Vidulić die Schlussfolgerung gezogen, dass Bahrs Befürwortung der föderalistischen Lösung, wie auch seine Sympathie für die Politik des `neuen Kurses in Dalmatien, auf einem fragmentarisierten und vereinfachten Verständnis der damaligen politischen Situation beruht. Dem Reisebericht lasse sich entnehmen, so Vidulić, dass das kroatische Volk für Bahr ein potentiell gehorsames Subjekt darstellte, das sich nur durch verfehlte Politik aus Wien zu einer zentrifugalen Kraft entwickelte.

Nachdem in mehreren Vorträgen die Produktivität der Raumforschung für die Analyse (post-)imperialer Narrative demonstriert wurde, zeigte Tatjana Jukić (Universität Zagreb) in ihrem Vortrag What Remains of Austria-Hungary: Psychoanalysis Imperial and Postimperial, dass dasselbe für die Psychoanalyse gilt. Jukić stellte die These auf, dass zwischen der 
strukturalen Logik der Donaumonarchie, die zum Ausbruch des Ersten Weltkriegs führte, und der Entwicklung der Psychoanalyse eine Korrelation bestehe. Als Beispiel für diese Korrelation wird Freuds Text Jenseits des Lustprinzips (1920) herangezogen. Wie bekannt, revidiert Freud in diesem Text seine Theorie von der metonymischen Topologie der Psyche, weil er anhand psychopathologischer Störungen bei Soldaten, die im Ersten Weltkrieg kämpften, die Unzulänglichkeiten seines analytischen Modells erkannte. Diese methodologische Intervention in das psychoanalytische Modell bezeichnete Jukić als das Ende der `imperialen Psychoanalyse‘, bzw. das Ende der metonymischen Logik der Territorialität, die schließlich zum Zusammenbruch der Monarchie führte. Diese These wurde unterstützt durch die Schriften von Victor Tausk, der die oben erwähnten psychopathologischen Störungen bei Soldaten dem Zustand >paranoia cum melancholia zuschreibt, obwohl Paranoia und Melancholie selten in Kombination auftauchen. Ihre Koexistenz ist nach Jukić kennzeichnend für den habsburgischen Kontext: Während Paranoia als Nebenwirkung ausgesprochener Subordination unter das paternalistische Prinzip entsteht, ist Melancholie eine Reaktion auf den extremen Verlust der Territorialität, den die Monarchie im Krieg erlitt. Beide Zustände sind sowohl bei den Soldaten als auch im Bezug auf die Monarchie erkennbar und führten, so der Schluss des Vortrages, zu den revolutionären Entwicklungen im Jahr 1914.

Während sich eine große Mehrheit der ReferentInnen auf den postimperialen Kontext oder den Übergang aus dem imperialen in den postimperialen Zustand unmittelbar nach dem Ersten Weltkrieg konzentrierte, widmete sich Wynfrid Kriegleder (Universität Wien) in seinem Vortrag Das Habsburger Imperium 1804-1825: Versuche seiner literarischen Legitimierung deutsch schreibenden Autoren der Habsburger Monarchie, die sich 1806, nach dem Zusammenbruch des Heiligen Römischen Reiches Deutscher Nation, in einer paradoxen Situation befanden: Obwohl sie sich sprachnational als Deutsche identifizierten, wurde von ihnen nicht das Deutsche, sondern das Habsburgerreich als der logische Nachfolger des untergegangenen Staates angesehen. In diesem Sinne wurde von Kriegleder das Epos Rudolf von Habsburg. Heldengedicht in zwölf Gesängen (1827) des Chefideologen des österreichischen Kaiserreichs Johann Ladislaus Pyrker untersucht, weil in diesem Werk das Grundproblem des österreichischen Kaisertums seit 1804 deutlich thematisiert wird: Wie kann die alte, übernationale Idee aus dem römischen Imperium mit dem neuen, österreichischen nationalen Bewusstsein in Verbindung gebracht werden?

Der erste Konferenztag wurde durch den Vortrag von Jelena Spreicer (Universität Zagreb) abgeschlossen. Spreicer stellte die historiografische 
Perspektive auf das Phänomen imperialer Zusammenbrüche in den Mittelpunkt. Wie bei einigen prominenten Historikern (Charles Tilly, Eric Hobsbawm, Alex Motyl u.a.) zu bemerken ist, werden imperiale Zusammenbrüche oft auf die den imperialen Strukturen inhärenten Pathologien zurückgeführt. Mit anderen Worten wird das Imperium als eine Form staatlicher Organisation angesehen, deren Dauer voraussichtlich begrenzt ist. Was jedoch gegen diese feste historiografische Überzeugung spricht, ist die Resistenz imperialer Staatsgebilde bis in die Gegenwart. Der Nationalstaat, der in Anlehnung an Müller-Funk als Gegenspieler des Imperialen bezeichnet werden könnte, ist im Vergleich zu Imperien ein relativ neues Konzept, das nichtsdestotrotz schon sehr früh tiefgreifende strukturelle Schwierigkeiten aufzuweisen hatte. Überdies wurde im Vortrag daran erinnert, dass prominente Theoretiker neuer Formen imperialer Herrschaft (David Harvey, Antonio Negri/ Michael Hardt u.a.) seit Jahrzehnten darauf hinweisen, dass imperiale Strukturen nur scheinbar zusammengebrochen sind. Diese Autoren konstatieren eine Wiederherstellung des Imperialen in Form von supranationalen und suprakontinentalen Epiphänomenen, die sich als resistent gegen innere Krisen erwiesen haben. Dementsprechend war es angebracht, den ersten Konferenztag mit der Frage abzuschließen: »Schlägt das Imperium tatsächlich zurück?»

Am 19. März wurde eine Reihe von Fallstudien vorgestellt. Das Thema des Vortrags von Jelena Šesnić (Universität Zagreb) unter dem Titel Images of America from the Austro-Hungarian Periphery war die Zeitperiode zwischen 1880 und dem Ausbruch des Ersten Weltkriegs, die durch massenhafte Emigration kroatischer Bevölkerung aus Österreich-Ungarn in die Vereinigten Staaten charakterisiert war. In dieser Periode ist eine Vielfalt von Texten entstanden, in denen kroatische Autoren (Skalica, Carić, Butković, Iveković, Sirotković, Hinković, Lupis-Vukić u.a.) die Begegnung mit der modernen amerikanischen Nation und Gesellschaft schildern. Šesnić konzentrierte sich auf den Reisebericht von Ante Tresić-Pavičić, Schriftsteller und Abgeordneter im Dalmatinischen Parlament, der 1906 nach Amerika reiste und im Jahr darauf seinen Reisebericht veröffentlichte. Vor der Folie postkolonialer Theorien (z.B. Mary Louise Pratt) wurde von Šesnić konstatiert, dass Tresić-Pavičićs Betrachtung der Vereinigten Staaten dem imperialistischen Diskurs entstammt. Trotzdem wird der imperiale Blick durch den Bedarf, die Aufmerksamkeit des Lesers ständig auf die dalmatinische und kroatische Subordination unter die österreichischen und ungarischen Machtzentren zu lenken, unterminiert. Die Überlegungen zur ökonomischen Situation der Kroaten in den Vereinigten Staaten werden abschließend als erstes Zeichen veränderter Machtverhältnisse auf der globalen politischen Szene gelesen. 
Einen weiteren wichtigen Beitrag lieferte Johanna Chovanec (Universität Wien), die weniger mit Überlegungen zur (post-)imperialen Situation der Doppelmonarchie aufwartete, sondern den Fokus auf das Osmanische Reich richtete. Ausgehend von Orhan Pamuk sprach sie im Vortrag Istanbul: eine melancholische Stadt im Kontext des >Osmanischen Mythos` über die Melancholie, die bei den türkischen Autoren nach dem Zerfall des Osmanischen Reichs vorkommt. Nachdem für die zeitgenössische türkische Literatur die Omnipräsenz osmanischer Geschichte festgestellt wurde, stellte Chovanec die Frage nach der Rolle der Stadt Istanbul in der Genese des so genannten ১Osmanischen Mythos $\lessdot$ Dieser wurde methodologisch in Anlehnung an Claudio Magris herausgearbeitet und als Folge der gebrochenen kulturellen Kontinuität in der Türkei nach der Gründung der Republik betrachtet. Die dadurch entstandene Entfremdung des Individuums von der Gegenwart wird in Pamuks Istanbul mit dem Wort `hüzun` (türkisch für Trauer und Melancholie) bezeichnet, wobei das Wort für Pamuk eine zusätzliche Bedeutung hat: die spezifische nostalgische Atmosphäre, die zum Kennzeichen der Stadt geworden ist. Aus diesem Grund sei, so resümiert Chovanec, gerade Istanbul als die zentrale Achse des `Osmanischen Mythos`zu betrachten.

Als Spezialistin für Finnougristik war Andrea Seidler (Universität Wien) die einzige Teilnehmerin, die ein hungarologisches Thema bearbeitete: die ungarische Leibgarde Maria Theresias, die aus jüngeren und älteren Mitgliedern des ungarischen Adels bestand und hauptsächlich im Kurierdienst der Kaiserin stand. Da die ungarischen Mitglieder sogar in dieser Garde, die eine ausschließlich repräsentative Rolle ausübte, keine Machtposition innehatten, zog Seidler die These von der Gleichstellung von Österreich und Ungarn in der Doppelmonarchie in Zweifel.

Die letzte Sektion leitete Milka Car (Universität Zagreb) mit einem Vortrag über die literarische Programmatik des kroatischen Autors August Šenoa ein. Aufgrund der Analyse seines literarischen Programms hinterfragte Car das homogenisierende nationale Narrativ, in dem Šenoa in einer ganzen Reihe kroatischer Literaturgeschichten einen festen Platz einnimmt. In diesem Sinne interessierte sich Car in erster Linie für die realistische Periode der kroatischen Literatur im 19. Jahrhundert, der wegen ihrer national orientierten und aufklärerisch-didaktischen Aufgabe von Aleksandar Flaker der Name >Protorealismus` zugeteilt wurde. Es wurde dabei eindeutig festgestellt, dass Šenoas Poetik trotz seines Status als erster kroatischer Romanautor zwischen nationalen und imperialen Interessen verankert ist.

Der Gegenwart am nächsten stand der Vortrag Habsburg und das Imperium 2016 von Johann Lughofer (Universität Ljubljana), in dem die 
gegenwärtige Wahrnehmung der Habsburger Monarchie untersucht wurde. Wie an einer Reihe von Beispielen (das Sissi-Museum, die Beerdigung von Otto von Habsburg u.a.) gezeigt wurde, erfreut sich die Dynastie im zeitgenössischen Österreich eines positiven Images. Für eine abschließende polemische Synthese sorgte Anna Babka (Universität Wien), indem sie die Notwendigkeit eines kritischen Zugangs zu (post-)imperialen Narrativen und die Unentbehrlichkeit der kritischen Theorie in der zeitgenössischen, vom postimperialen Erbe nach wie vor belasteten Gesellschaft unterstrich.

Die Bilanz des Workshops ließe sich folgendermaßen formulieren: Erstens weist die methodologische und thematische Breite der vorgestellten Forschungsresultate auf die Ergänzungsbedürftigkeit wissenschaftlicher Erkenntnisse im Forschungsfeld >postimperiale Narrative hin. Zweitens ist nach dem ersten Projektjahr ein beachtliches Korpus von Beiträgen entstanden, so dass in einem nächsten Schritt die Publikation der Ergebnisse geplant wird. Und drittens, dem Forschungsteam steht in den nächsten drei Projektjahren eine intensive Arbeit an den hier präsentierten Forschungsthemen bevor. 


\section{ZAGREBER} GERMANISTISCHE BEITRÄGE
ISSN 1330-0946 (print)

ISSN 1849-1766 (online)

CODEN ZGBEEY

UDK $803.0+830$

Das Jahrbuch Zagreber Germanistische Beiträge veröffentlicht wissenschaftliche Aufsätze, Berichte und Buchbesprechungen in den Bereichen: Literaturwissenschaft, Sprachwissenschaft, Kulturwissenschaft, Übersetzungswissenschaft und Didaktik des fremdsprachlichen Deutschunterrichts.

ZGB erscheint seit 1992 in gedruckter Form.

ZGB ist ab Nr. 1 (1992) zugänglich in der Central and Eastern European Online Library (C.E.E.O.L) unter <http://www.ceeol.com>.

ZGB ist ab Nr. 20 (2011) frei zugänglich beim Zeitschriftenportal Hrčak - portal znanstvenih časopisa Republike Hrvatske unter <http://hrcak.srce.hr/zgb>.

ZGB wird außerdem in folgenden Datenbanken gelistet: Bibliographie der deutschen Sprach- und Literaturwissenschaft (BDSL); EBSCOhost; European Reference Index for the Humanities and Social Sciences (ERIH PLUS); Linguistic and Language Behavior Abstracts (LLBA ); Linguistic Bibliography Online; MLA International Bibliography und MLA Directory of Periodicals.

\section{Herausgeber / Izdavač}

Abteilung für Germanistik der Philosophischen Fakultät der Universität Zagreb

\section{Schriftleitung / Uredništvo}

Maja Anđel (Sprachwissenschaft), Marijan Bobinac (Literaturwissenschaft), Milka Car (Literaturwissenschaft), Svjetlan Lacko Vidulić (verantw. Chefredakteur), Marija Lütze-Miculinić (Sprachdidaktik), Slađan Turković (Sprachwissenschaft)

\section{Wissenschaftlicher Beirat / Uredničko vijeće}

Daniel Baric (Paris), Hans Richard Brittnacher (Berlin), Zrinjka Glovacki-Bernardi (Zagreb), Mirko Gojmerac (Zagreb), Maja Häusler (Zagreb), Dragutin Horvat (Zagreb), Hubert Lengauer (Klagenfurt), Irmela von der Lühe (Berlin), Helga Mitterbauer (Bruxelles), Wolfgang Müller-Funk (Wien), Walter Pape (Köln), Velimir Piškorec (Zagreb), Boris Previšić (Luzern), Ivo Runtić (Zagreb), Hannes Scheutz (Salzburg), Stanko Žepić (Zagreb), Viktor Žmegač (Zagreb)

\section{Sekretariat / Tajništvo}

Monika Blagus, Jelena Spreicer; Lektur: Yvonne Jock, Stephan Kurz, Svjetlan Lacko Vidulić

\section{Hinweise für Verf.}

Sprache: Beiträge in deutscher Sprache, Zusammenfassung (bis 700 Zeichen) und Schlagwörter (3-5) bitte in deutscher und englischer Sprache. Lektorat:

Eingereichte Beiträge, die eine umfassende Lektur benötigen, gehen an die Verfasser mit der Bitte um Nachbesserung zurück. Begutachtung: Jeder Beitrag wird von zwei FachexpertInnen anonym begutachtet. Für Beiträge von Mitgliedern des Instituts, das als Hg. der ZGB zeichnet, werden die Gutachten ausnahmslos aus dem Ausland eingeholt. 